\title{
Synthesis of Nanomaterials and Nanocomposites with Complex Shapes through Arc Discharge in Foam*
}

\author{
Yong il Kim, ${ }^{\dagger}$ Eiichi Nishikawa, and Masatoshi Sano \\ Division of Electrical Engineering, Graduate School of Tokyo University of Science, 162-8601 Tokyo, Japan
}

(Received 8 November 2008; Accepted 30 January 2009; Published 21 March 2009)

\begin{abstract}
Several types of carbon nanomaterial were produced using a simple method involving arc discharge in foam, and Fe-C composite materials were produced using this technique in foam containing Fe particles. In TEM observation, it was observed that the surfaces of the Fe particles were covered with a 20-50 carbon layer. Spherical objects with complex shapes were also observed on the nanomaterials, and varied from $200 \mathrm{~nm}$ to above $600 \mathrm{~nm}$ in size. It was found that these spherical objects formed a twisted graphite sheet. [DOI: 10.1380/ejssnt.2009.195]
\end{abstract}

Keywords: Carbon nanomaterials; TEM; Arc discharge; Foam

\section{INTRODUCTION}

The discovery of nanomaterials such as fullerene $\left(\mathrm{C}_{60}\right)$ [1], carbon nanotubes (CNTs) [2], nanoparticles with an onion-like structure of concentric atomic carbon layers [3] and several types of nanocomposite $[4,5]$ has created new fields of material research and development.

In recent years, the study of nano-sized materials such as nanocomposites has attracted considerable interest due to their usefulness in microelectronic devices, metallurgy, surface modification, catalysis, biological labeling, photonics and information storage [6-8].

In particular, the technique by which nanomaterials are coated is a key technology in drug delivery systems (DDS), and the release of active components in drugs is controlled by the selection of the coating material and the thickness of the coating layer. To apply nanomaterials to practical devices, it is important to have a simple method of synthesis for nanocomposites.

In our previous study, we investigated the production of CNTs using the DC arc plasma method in foam. The merits of this method include the low cost and ease of nanomaterial production as compared with synthesis in a vacuum. During the investigation of arc plasma processing, we observed a phenomenon that allowed the use of arc plasma irradiation to coat fine Fe particles with a thin carbon film. We also noted that the fine particles attached to the foam surface were spherical objects with a complex-shaped cross section.

The main purpose of this study was to establish a simple method of preparing several types of nanomaterial using arc plasma irradiation. We conducted a study from the unique point of view of creating a new method to synthesize nanomaterials using arc discharge with the aim of producing several types of nanomaterial at low cost.

\section{EXPERIMENTAL}

Figure 1 shows a schematic diagram of the equipment used to produce Fe-C nanocomposite materials using the arc plasma method in foam. Regarding the environment

\footnotetext{
* This paper was presented at International Symposium on Surface Science and Nanotechnology (ISSS-5), Waseda University, Japan, 9-13 November, 2008.

†Corresponding author: j4306702@ed.kagu.tus.ac.jp
}

of the arc discharge, we used foam generated from a beer dispenser (Fukushima Industries Corporation: FTN$38 \mathrm{~S} 1$ ) as the beer froth supply, and the pressure of the carbon dioxide gas in the dispenser was set to $2.4 \mathrm{~kg} / \mathrm{cm}^{2}$. Fe particles with an average diameter of between 0.2 and 0.5 micrometer were used as the core particles for the coating process.

The overall system essentially consisted of a glass container filled with foam, graphite electrodes for the anode and cathode, and a DC power supply. This simplified design has many advantages, including elimination of the need for gas purging and the absence of complicated vacuum seals.

The process was as follows: Fe particles were arranged at the bottom of the glass container. When beer is poured into a glass container from a beer dispenser, the input of mechanical energy causes gas breakout from supersaturated solution and the bubbles formed rise to the surface. The resulting foam, containing the Fe particles on top of the glass container, be fine bubbled and stable. Stable foam is a characteristic of beer. The glass container should always be absolutely clean so that grease on glass container does not cause premature foam collapse. Furthermore, the stability of the foam was ensured by con-

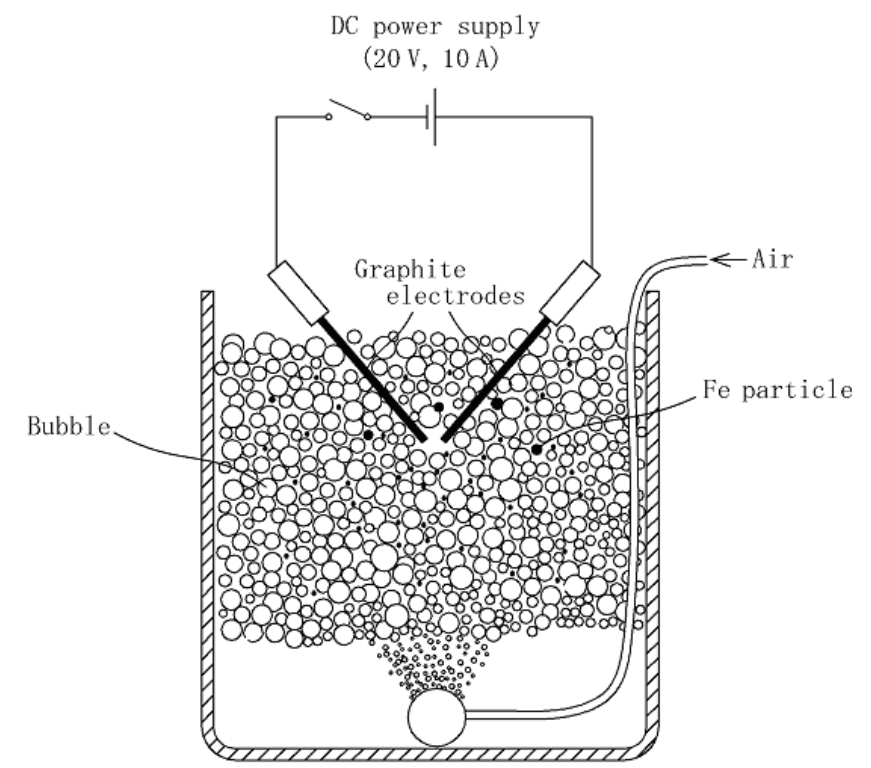

FIG. 1: Schematic diagram of the equipment used for producing fine composite particles using the arc plasma method. 

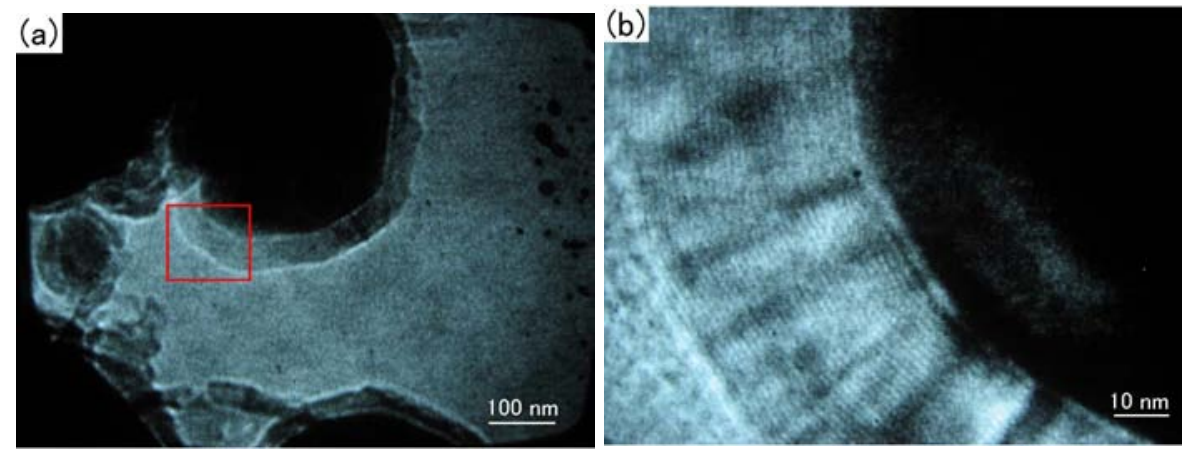

FIG. 2: HR-TEM micrographs of the Fe-C nano composite:

(a) Under low magnification; (b) Highly magnified views of (a).
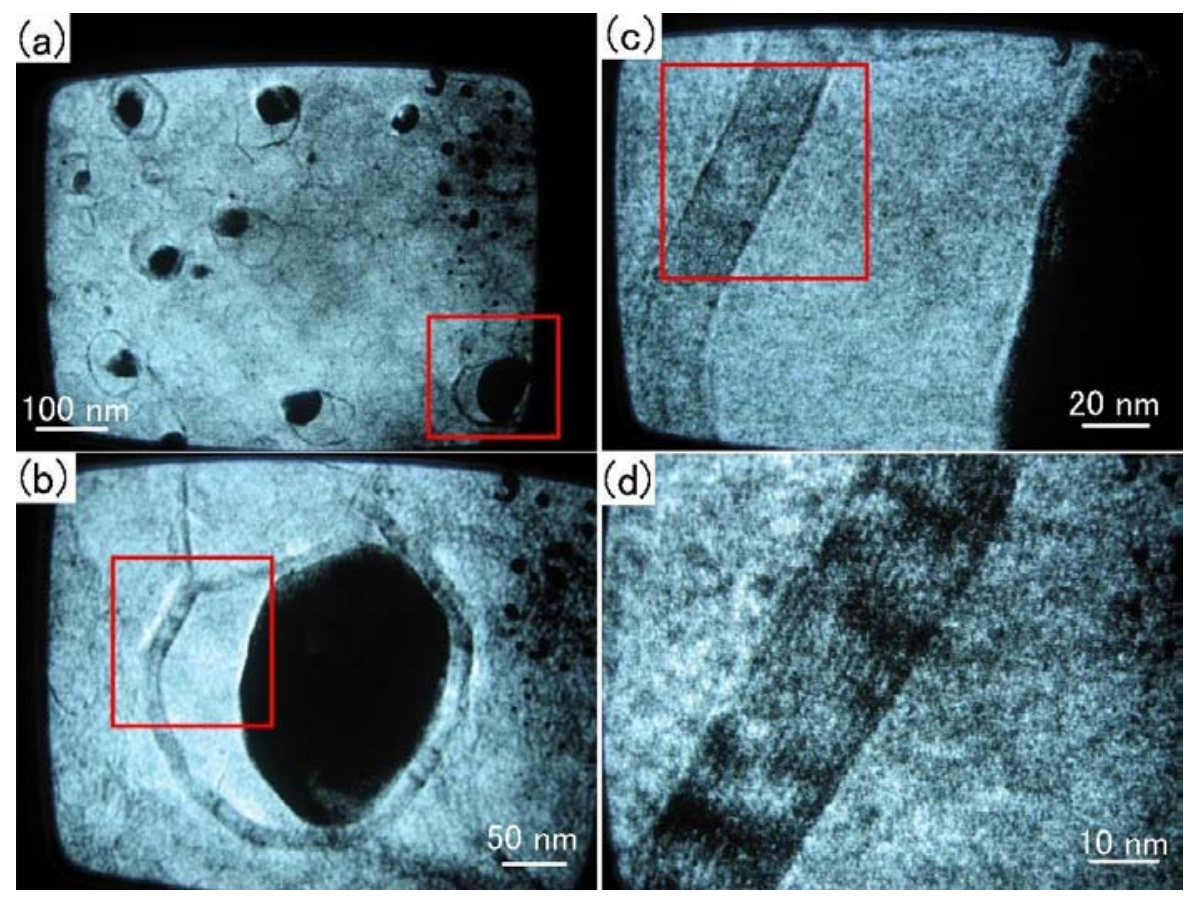

FIG. 3: HR-TEM micrographs of the Fe-C nano composite: views of (a).

(a) Under low magnification; (b), (c) and (d) Highly magnified

tinuously supplying air through an air pump.

Both the graphite electrodes were dipped into the foamfilled open container. They were momentarily brought into contact and an arc discharge was initiated. During this process, the space in which the closed arc discharge existed consisted of foam; the discharge voltage and current were optimized and maintained at $20 \mathrm{~V}$ and $10 \mathrm{~A}$, respectively, and the synthesis time was $1 \mathrm{~min}$. We also generated fine particles through arc discharge in simple foam without Fe particles.

The reason that we choose the beer in the foam is that the bubble has the properties both of liquid and gas. Therefore, the bubble offers ideal sealing environment for arc discharge. Because the froth can be prepared using surfactants which are primarily proteins in the case of beer, the frothing ingredients create viscous membranes. In addition, although the surface of the froth swells due to the arc discharge, its surface tension does not allow the foam on the surface to break.

As a result, it is possible to confine the arc discharge to the space within the froth, whereby we induced a DC arc discharge lasting for several minutes.

After the arc discharge, countless fine particles were trapped on the froth surface. We placed some of these particles on a microscope specimen grid and examined them under a high-resolution transmission electron microscopy (HR-TEM) system operating at $200 \mathrm{kV}$ (HR-TEM model H9000; Hitachi Co., Ltd.).

\section{RESULTS AND DISCUSSION}

Figures 2 and 3 show typical TEM images of the Fe-C composite materials generated through arc discharge in foam with $\mathrm{Fe}$ particles. Figure 2(b) shows an enlarged view of (a). The shape and size of the Fe-C nanocomposite materials were determined from the TEM micrographs, which show a well-crystallized straight carbon 


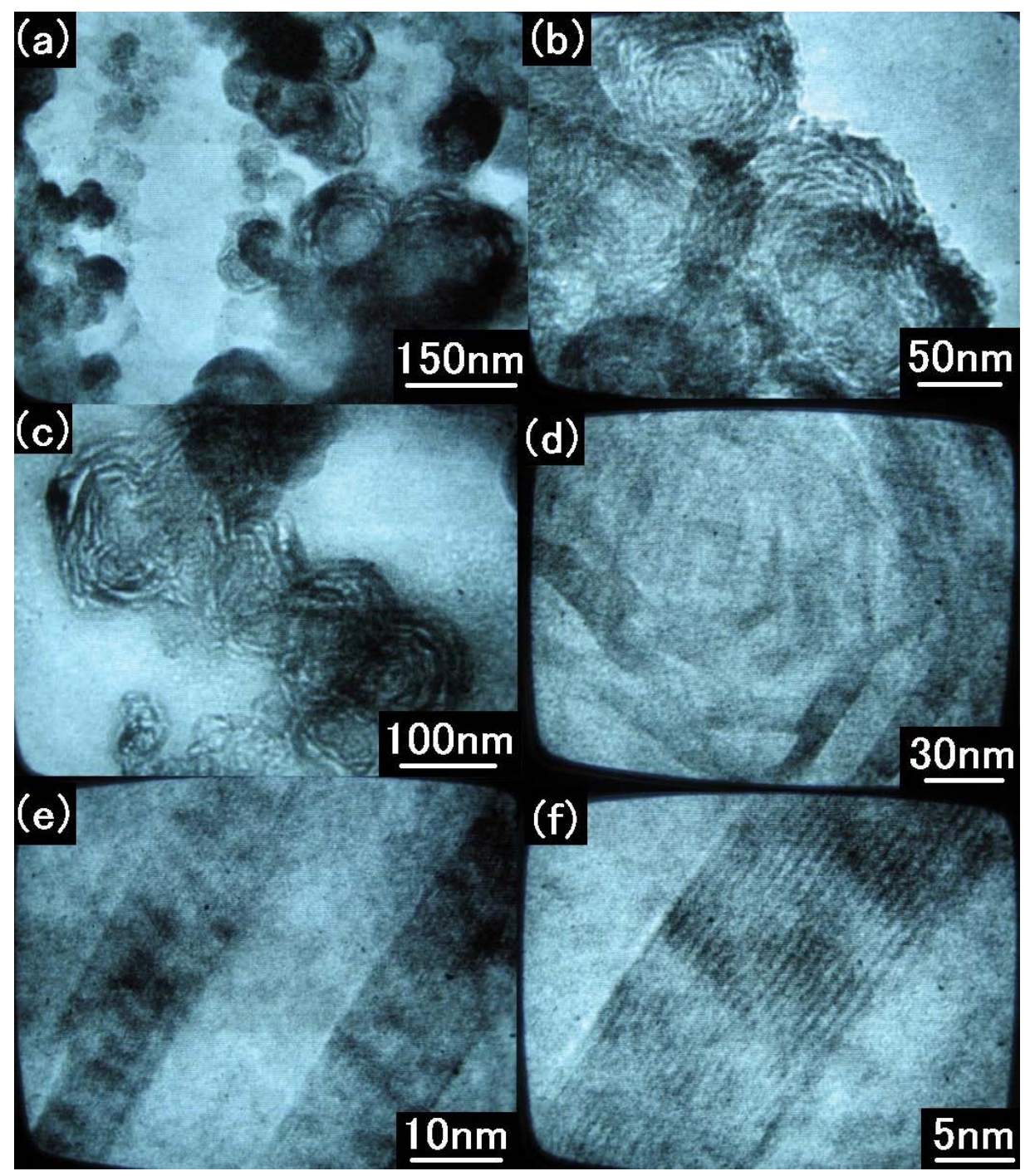

FIG. 4: HR-TEM micrographs of the complex-shaped nanomaterial: (a)-(c) Under low magnification; (d)-(f) Highly magnified views.

layer around the fine Fe particles. The Fe-C composite materials typically had a diameter of $200 \mathrm{~nm}$ and consisted of 40 carbon layers; the diameters of the inner shells of the Fe particles were approximately $150 \mathrm{~nm}$.

The TEM micrographs provided some valuable information about the quality of the Fe-C composite materials produced: the $\mathrm{Fe}$ particles exhibit a microspheric form with a smooth surface, which is covered with a 20-50 carbon layer. These layers were of a high quality, consisting of long, straight parallel walls with only occasional surface contamination.

Figures 3(a)-(d) show typical TEM images of the Fe-C composite materials produced; Figures $3(\mathrm{~b})-(\mathrm{d})$ show enlarged views of (a). The space between an Fe particle and the carbon layer was observed (Figs. 3(b)-(d)), revealing that the particle was wrapped in a capsule consisting of 20 layers of carbon. Thus, two different shapes were seen, as shown in Fig. 2 and Fig. 3.

In general, the temperature of a plasma arc is known to exceed $10,000^{\circ} \mathrm{C}[9]$. Under such conditions, there are several possible mechanisms leading to the formation of Fe-C composite materials.
One of these is the evaporation of $\mathrm{C}$ on the cathode surface. Carbon molecules made to evaporate by the arc discharge cover the surfaces of the Fe particles. Another mechanism is the evaporation of Fe particles. The melting temperature of $\mathrm{C}$ is much higher than that of Fe. Solid $\mathrm{Fe}$ particles become $\mathrm{Fe}$ steam at around $1,500^{\circ} \mathrm{C}$, and $\mathrm{Fe}$ particles come in contact with the carbon layer at one point. The Fe particles therefore serve as a catalyst and react with the carbon molecules.

Figures 4(a)-(f) show HR-TEM images of the fine particles generated by arc discharge in simple foam without Fe particles. In these TEM observations, these spherical objects were in fact found to form a twisted graphite sheet with a complex shape.

It was also determined that the internal structure consisted of graphite sheets with a diameter of $20 \mathrm{~nm}$ folded into a multi-angle structure with a complex-shaped cross section, as shown in Figs. 4(e)-(f). This material was 10$50 \mathrm{~nm}$ in thickness and consisted of roughly 20-100 layers of graphite sheet. The space between adjacent sheets produced a cabbage-shaped cross section, as shown in Fig. $4(\mathrm{~d})$. 
The diameter of these complex-shaped nanomaterials varied from $200 \mathrm{~nm}$ to more than $600 \mathrm{~nm}$, and they were grouped into agglomerations consisting of several dozen to several hundred particles. Additionally, although nanotubes and nano-onions were not observed in the groups of complex-shaped nanomaterials, they were found to be mixed together with nano-onions and fullerenes. Since nanotubes are usually observed as one-dimensional needle-like objects, and nano-onions and fullerenes extend radially, their structure is different from that of complexshaped nanomaterials.

It is assumed that complex-shaped nanomaterials are formed when carbon vapor evaporated as a result of the arc discharge becomes entrapped inside fine foam and is subsequently transformed into graphite as it shrinks and cools together with the foam.

It is also thought that these nanomaterials appear in agglomerations due to the aggregating effect of the foam.

However, beer constituents comprise more than 800 compounds [10], also there are effect of ethanol on the foaming properties of beer protein [11]. So, it is difficult to specify the materials and molecules of the foam, at present, no confirmation of the formation mechanism has been made, and this is a task for future studies.

\section{CONCLUSIONS}

In summary, we have proposed a method of synthesizing Fe-C composite materials and complex-shaped nanomaterials using the arc discharge method in foam. The Fe-C composite produced (Fe particles covered with a 20-50 carbon layer) was observed, and a uniform carbon coating was found to have been successfully produced on singlecore Fe particles using the simple DC arc method. We have also reported on the generation of complex-shaped nanomaterials as a result of arc discharge in foam.

The structure of these nanomaterials consists of graphite sheets twisted in a complicated way, and it can be expected that the strength of nano-sized springs may be further improved by the application of this material. Moreover, these nanomaterials are created as a result of arc discharge in foam, and can be easily collected as fine particles attached to the surface of the foam. In addition, the internal structure of this material consists of alternately folded graphite sheets. Since the characteristics of such a structure enable the encapsulation or absorption of different molecules or DNA, the use of these nanomaterials as hydrogen adsorption agents or for drug delivery is expected.

From this study, we conclude that the proposed method represents a simple, efficient technique for the fabrication of several types of nano-sized composite materials, and is useful for designing materials with vastly different sets of properties.

\section{Acknowledgments}

This work was supported by the Center for Drug Delivery Research (DDS) at Tokyo University of Science, Japan.
[1] H. W. Kroto, J. R. Heath, S. C. O'Brien, R. F. Curl, and R. E. Smalley, Nature 318, 162 (1985).

[2] S. Iijima, Nature 354, 56 (1991).

[3] N. Sano, H. Wang, M. Chhowalla, I. Alexandrou, and G. A. J. Amaratunga, Nature 414, 506 (2001).

[4] A. Tsutsumi, M. Ikeda, W. Chen, and J. Iwatsuki, Powder Technology 138, 211 (2003).

[5] T. Nakagawa, M. Hieda, D. Shindo, F. Saitoa, K. Yubuta, K. Nishimura, and T. Fujii, Powder Technology 130, 456 (2003).

[6] Y. I. Petrov, Clusters and Small Particles (Nauka, Moscow, 1986) p. 367 (in Russian).

[7] J. M. Capus, Powder Metall. 46, 8 (2003).
[8] I. Zalite, S. Ordanyan, and G. Korb, Powder Metall. 46, 143 (2003).

[9] M. Uda, Bull. Jpn. Inst. Met. 22, 412 (1983) (in Japanese).

[10] S. Cortacero-Ramirez, M. Hernainz-Bermudez de Castro, A. Segura-Carretero, C. Cruces-Blanco, and A. Fernandez-Gutierrez, Trends in Analytical Chemistry 22, 440 (2003).

[11] E. R. Brierley, P. J. Wilde, A. Onishi, P. S. Hughes, W. J. Simpson, and D. C. Clark, J. Sci. Food Agric. 70, 531(1999). 